\title{
Diseño e Implementación de un Modelo de Gestión en Docencia Universitaria: Caso Carreras de Ingeniería de la Universidad Arturo Prat, Chile
}

\author{
Marianela Llanos y Alberto A. Martínez \\ Facultad de Ingeniería y Arquitectura, Universidad Arturo Prat, Avda. Arturo Prat № 2120, Iquique-Chile. \\ (e-mail: mllanos@unap.cl; albmarti@unap.cl)
}

Recibido Abr. 18, 2018; Aceptado Jun. 13, 2018; Versión final Jul. 4, 2018, Publicado Dic. 2018

\begin{abstract}
Resumen
Se propone un modelo para la modernización de la Gestión en Docencia Universitaria. Se analiza el caso de las carreras de Ingeniería de la Facultad de Ingeniería y Arquitectura, Universidad Arturo Prat. Históricamente estas carreras habían alcanzado bajo desempeño en indicadores de reprobación, deserción y titulación oportuna, afectando sus procesos de acreditación desde el 2011. Lo anterior motiva una revisión en las carreras, para realizar seguimiento e intervenciones oportunas desde la cohorte 2013. Resultado, es el diseño e implementación de un Modelo de Gestión Docente, con bases en Análisis Modular de Sistemas, Gestión del Conocimiento, Sistémica Cibernética y Sociológica. El Modelo propuesto representa tres Módulos (ámbitos), de repercusión decisional: (1) De Entrada: Selección y Matrícula, (2) De Proceso: Aprobación y Deserción y (3) De Salida: Titulación y Empleabilidad. Su implementación genera incremento de 14 puntos porcentuales en Retención y 21 puntos porcentuales en Aprobación de primer año al 2016.
\end{abstract}

Palabras clave: gestión docente; progresión del estudiante; cibernética; autoorganización.

\section{Design and Implementation of a Management Model in University Teaching: Case of Engineering Programs at Arturo Prat University, Chile.}

\begin{abstract}
A model is proposed for the modernization of graduate education management. The case of the Engineering Programs of the Faculty of Engineering and Architecture of the Arturo Prat University is analyzed. Historically, these programs reached low performance in reprobation, desertion, and timely graduation indicators, affecting appropriate monitoring and intervention processes since 2013 student cohort. The result is a design and execution of an Educational Management Model, with basis in System modular analysis, knowledge management, Cybernetic and Sociological Systemic. The proposed model represents three modules (areas) of decisional repercussion: (1) Input: Selection and registration, (2) Processing: Approbation and Desertion and Output: Graduation and Employment. Its execution generates a 14 percent increase in retention and 21 percent increase in freshmen approbation at 2016.
\end{abstract}

Keywords: educational management; student progression; cybernetic; self-organization 


\section{INTRODUCCIÓN}

Estudios realizados sobre el Panorama de la Educación Superior en Chile (SIES, 2015), por la División de Educación Superior del Ministerio de Educación, dan cuenta de la expansión de la matrícula y diversicación institucional de la educación superior chilena desde la década de los 80 , alcanzando procesos de estabilización, consolidación y transformación interna. Este desarrollo trae consigo una serie de efectos sistémicos y, a la vez, nuevos desafíos como el cambio en el perfil de los estudiantes, es decir, la masificación implica la inclusión de estudiantes no tradicionales, provienen de sectores más vulnerables, con una preparación académica muchas veces deficiente, y un grupo importante requiere compatibilizar estudios y trabajo. Estas nuevas condiciones implican nuevos retos para las instituciones, que dicen relación con favorecer el acceso con equidad, evitar la deserción, desarrollar iniciativas de nivelación y asegurar modalidades flexibles y adecuadas para el nuevo tipo de estudiantes, manteniendo los niveles de calidad (Tinto, 2007)

En base a estudios entregados por el Servicio de Información de Educación Superior (SIES), una problemática transversal es la falta de eficacia y eficiencia en los procesos formativos de la educación superior en Chile, que demandan recursos del estado y de los propios individuos, con resultados de reprobación y deserción poco eficientes. Esto se condice con la información disponible de las cohortes 2008-2012 a nivel nacional. Otro hallazgo, es que las instituciones de Educación Superior acreditadas, tienen persistentemente mayor tasa de retención de 1er año, que aquellas instituciones que no lo están. En particular para el año 2014, las instituciones acreditadas alcanzaron una tasa de $72,2 \%$ y las instituciones sin acreditación un $53,7 \%$. Esta brecha se ha acentuado ampliamente los últimos 3 años abordados por el estudio, llegando el 2014 casi a los 20 puntos. El panorama regional muestra que para la cohorte 2014, las regiones que lideran las menores tasas de retención se dan en las regiones más extremas: Atacama (57,5\%), Tarapacá (62,3\%), Antofagasta (64,8\%), Aysén $(66,9 \%)$ y la región de Arica y Parinacota $(67,8 \%)$. (SIES, 2015). Esta deserción, tiene un impacto social e individual relevante para el desarrollo del país, costos elevados de formación sin término, frustración y endeudamiento familiar, sobre la expectativa de lograr una mejor calidad de vida y estatus. Chile es uno de los países donde más aportan las familias a la educación superior, el país destina 2,1 \% del PIB, de los cuales 0,3 puntos porcentuales se originan en financiamiento público y 1,8 puntos porcentuales en financiamiento privado.

En los últimos años, el Estado ha promovido, a través de diversos instrumentos, la formulación de estrategias de retención de estudiantes, como el Programa de Mejoramiento de la Calidad y Equidad en la Educación Superior (Mecesup), el cual ha intentado instalar la preocupación por la deserción en las instituciones, financiando acciones y programas para nivelar las oportunidades de aprendizaje de los estudiantes, y fomentar su permanencia en el sistema (Zapata, 2016). Asimismo, el SIES retroalimenta periódicamente al entorno de educación superior chileno, con el objetivo de contribuir a las decisiones institucionales y a la política pública en esta materia. En este escenario, las Universidades chilenas deben conciliar los desafíos de viabilidad económica con su compromiso de responsabilidad social, en ambientes cambiantes, con demandas que emergen de una sociedad cada vez más compleja, dinámica y competitiva. Otra exigencia es la internalización de una cultura de indicadores de proceso y resultados, lo que se ha ido consolidado en la última década, y seguirá evolucionando hacia nuevos indicadores, para instalarse en los mecanismos de aseguramiento de la calidad del sistema de educación superior chileno.

Uno de los hechos que empieza a ser recurrente, es la necesidad de desarrollar modelos de gestión docente, acorde a estos desafíos que absorban la complejidad y que permitan orientar los esfuerzos de los cuerpos directivos, para el logro de objetivos estratégicos que deben plantearse las universidades, en un contexto dinámico e incierto. Este trabajo se enmarca en la necesidad de responder a estas interrogantes, para contribuir a la modernización del modelo de gestión en Docencia Universitaria en Chile. Para ello, es importante incorporar en el mundo académico, el concepto de gestión, en una perspectiva moderna hacia Universidades Inteligentes, con capacidades de aprender (Barnett 2002), es decir, que la gestión bajo condiciones de extremas turbulencias del entorno, requiere habilidades totalmente diferentes, en especial para aceptar la dinámica y la complejidad; permitir y equilibrar las contradicciones y las paradojas; ejercer el pensamiento en coherencia con el actuar sistémico, implica conducir, potenciando las sinergias para facilitar el aprendizaje organizacional o la generación de conocimiento. Entendiendo que cuando la información es interpretada y se le da un contexto, se convierte en conocimiento (Nonaka, 2006).

El paradigma de la complejidad, aplicado al gobierno de las organizaciones, parte del supuesto de que el mundo es tan interdependiente, que nada es totalmente predecible, Ashby demostró que únicamente sistemas con un alto grado de variedad interna pueden procesar un alto grado de variedad del entorno, lo que se conoce como ley de requisito de variedad: "solo la variedad absorbe la variedad" (Asbhy, 1961). Ello quiere decir que las organizaciones con estructuras diseñadas desde paradigmas más avanzados, como el sistémico-cibernético y complejo, estarán mejor preparadas para abordar de modo efectivo las tareas que 
demandan los ambientes de incertidumbre (Espejo, 2011). Las Universidades chilenas se encuentran en una etapa de transición, presionadas por exigencias de las acreditaciones, que responden a un modelo para evaluar Calidad de la Docencia Superior (Zapata, 2016), incorporando a su gestión una visión holística, con desarrollo de Planes Estratégicos e incorporando las tecnologías de la información, para la generación de información oportuna, pero con una cultura que aún no se sintoniza con las nuevas propuestas. En la búsqueda de cómo abordar los cambios, se visibiliza la Gestión del Conocimiento, que precisa un tiempo de aprendizaje, la consolidación de lo aprendido y la creación de la habilidad necesaria para obtener el máximo partido a los conocimientos asimilados. Para la distribución del conocimiento existen facilitadores como la tecnología, las personas y la cultura organizacional que deben gestionarse adecuadamente para su distribución y afianzamiento del conocimiento. La tecnología por sí misma no es suficiente, se requiere promover el trabajo en equipo, la colaboración y la creación de espacios para la reflexión y el aprendizaje social (Senge, 2001).

El aproximar modelos de gestión con las características planteadas, requiere de la Cibernética que tiene relación con el control y la comunicación en sistemas vivos, en máquinas y en estructuras organizadas. El enfoque de Caja Negra, que es uno de los métodos usados para hacer frente a la complejidad, operando como un reductor de ella, en una lógica donde lo que puede decir del sistema, es extraído de la información que se recoge de los cambios de estados observados en la salida, conforme a los estímulos entregados a la entrada por el administrador. La Caja Negra o Módulo se puede intervenir o abrir, identificando en el mapa recursivo su posición, para restablecer las referencias definidas por el administrador de ella (Asbhy, 1961).

El Análisis Modular de Sistemas asegura la transición de la estrategia y el nivel de la estructura, a la gestión de niveles, el control de gestión y el nivel de información. Por lo tanto, se presenta como una metodología de descripción de la organización que el administrador pueda usar para comprender anomalías o problemas y formular las acciones correctivas, que pueden ser referidos a la estructura, en cuanto a dominios de la administración o la informática (Mélèse, 1991). Esto implica que su utilidad se orienta en el diseño de sistemas de información, la rehabilitación de la función de la organización frente a la información, la comunicación entre todos los actores afectados por un cambio. Entendiendo la complejidad de la organización, como un aumento de las interacciones entre las diferentes unidades básicas o los diferentes centros de decisión. Esto estimula la acción reflexiva de introducirse en la observación del observador, que se vuelve relevante en el dominio de la toma de decisiones. El objeto de estudio pasa a constituirse en el observador, por lo que el conocimiento pasa a ser inseparable de la acción (Nonaka, 2006). Esto pertenece a la cibernética de segundo orden, abriendo un espacio para la reflexión sobre el propio comportamiento y entra directamente en el territorio de la responsabilidad y la ética (Espejo, 1993). Dado que se fundamenta en la premisa de que no somos descubridores de un mundo exterior a nosotros, sino inventores o constructores de la propia realidad, todos y cada uno de nosotros somos fundamentalmente responsables de nuestras propias invenciones (Maturana y Varela, 2006).

A medida que aumenta la complejidad del sistema y la dinámica del entorno, que es lo que enfrentan las universidades chilenas, crece la necesidad de reemplazar la visión tradicional con estructura jerárquica, hacia una aproximación estructural y funcional (Espejo, 2004; Tarride, 2008). La concepción de la organización como un "ente vivo" (Beer, 1997), basado en el estudio del sistema nervioso humano, permite avanzar en el diseño organizacional, al incorporar conceptos de la biología en la administración, inspirando a distintos autores nacionales e internacionales que plantean propuestas y reflexiones con este nuevo enfoque como: Gareth Morgan, Frijof Capra, Peter Senge, Margareth Wheatley, Ikujiro Nonaka, Arie de Gens, Raúl Espejo, Darío Rodríguez, Marcelo Arnold y Humberto Maturana. Los distintos autores mencionados concuerdan, que una de las principales características de los sistemas complejos es la autoorganización, la que les permite transformarse creando estructuras altamente organizadas que pueden transformar su entorno. Esta capacidad les otorga, a diferencia de las máquinas, la posibilidad de adaptarse a los cambios que ocurren en el entorno o medio (Morin, 2004). Para comprender esta complejidad se debe distinguir que la organización como todos los sistemas tienen existencia simultánea en dos dominios: Aquel en que se comporta como unidad simple y aquel en el que funciona como unidad compuesta. Así, la organización se manifiesta en el dominio físico, en el que existimos como seres vivos y en el dominio o espacio social donde existimos como personas, compartiendo un dominio generado por nuestras interacciones con el resto de los seres humanos.

El dominio social (con el surgimiento del lenguaje), que coordina las conductas de los seres humanos se puede explicar desde la teoría de la Biología del Conocimiento, de los autores chilenos Maturana y Varela, re interpretando las organizaciones como sistemas de actividad humana con propósito productivo, las cuales, se constituyen en la relación humana y permiten acercar la comprensión de lo humano para el diseño organizacional. Maturana al explicar el fenómeno del conocer, parte de la experiencia del observar y se pregunta por el origen de las capacidades del observador. En el proceso de responder a esta pregunta desarrolla lo que el autor denomina "La ontología del observar" (Maturana, 1990). En este proceso se muestra que el explicar científico no hace referencia a una realidad independiente del observador y que no se requiere 
el supuesto de una tal realidad, postulando que la noción de realidad es una proposición explicativa. Así dice: "Todo lo dicho es dicho por un observador a otro observador que puede ser él o ella misma en su propuesta de suspender la convicción respecto a la objetividad de nuestras percepciones, invitando a poner la objetividad entre paréntesis en el proceso del explicar" (Maturana, 2004). Para Nonaka, la gente aprende y crea conocimiento a través de interacciones sociales continuas, este autor, propone un modelo creador de conocimiento a través de una teoría cognitiva de la organización, en la que la empresa crea conocimiento a través de la síntesis de la subjetividad y la objetividad en el proceso SECI de conversión del conocimiento. Lo hace a través de Diálogos y prácticas. El proceso cuenta con una Visión cognitiva y Objetivos impulsores, los cuales dan dirección y energía al proceso SECI en un contexto compartido (Ba) en movimiento, el cual provee un lugar experiencial con Recursos cognitivos como insumos y resultados del proceso SECI, a través de la interacción con el Entorno (ecosistema) de conocimiento y Ba de varios niveles (Nonaka, 2006). Todas estas distinciones permiten comprender y aproximarse de mejor forma al proceso de toma de decisiones en las organizaciones y la generación de conocimiento en una espiral de procesos de aprendizajes, innovación y cambio.

Por otro lado, la autopoiesis, es una dinámica operacional que se manifiesta mediante una red cerrada de relaciones de producción de componentes; los que al interactuar entre sí dan como resultado la configuración de los mismos procesos que los generaron a ellos. Así, la organización puede ser definida: "Como un conjunto de actos laborales, tecnológicos y económicos en clausura operacional que constituyen a la empresa como una unidad en el espacio social" (Rodríguez, 2003). Las personas no constituyen la empresa, sino que la producen; y la producen ininterrumpidamente, pues si dejaran de producirla por un espacio de tiempo no soportable por el sistema, la empresa se desintegraría. En síntesis, estas teorías permiten estudiar la organización humana como un sistema que se hace a sí mismo continuamente, para mantener su organización. El mecanismo que permite esto, es una red de procesos bastante similar al de la autopoiesis, pero que difiere con este en las actividades específicas. Ese proceso de autogeneración de la organización humana ha sido llamada Sociopoiesis (Limone, 2002; Arnold et al., 2011).

Las teorías sistémico cibernética y sistémico sociológica (Limone, 2002; Espejo, 2004; Arnold et al., 2011), abordan en conjunto la tarea de dar una explicación más amplia, profunda y conectiva, para poder tratar a las organizaciones humanas como sistemas complejos adaptativos. Sus características centrales del sistema "organización humana y empresarial" que la fundamentan son: (1) La generación del sistema se realiza a través del establecimiento de tres relaciones constituyentes: Las relaciones de especificación, las constitutivas o topológicas y las de orden. (Maturana y Varela, 2006).; (2) En el sistema generado de esta forma es posible distinguir tres grandes procesos o macro-procesos que operan en clausura operacional (Maturana, 1990), esto es, con una dinámica organizacional/operativa cerrada. En relación a sus intercambios con el medio, el sistema es abierto. Los macro-procesos son los siguientes: (a) El proceso primario, que es un proceso de naturaleza financiera y comportamiento cibernético que permite la regeneración continua de los fondos utilizados en la mantención de la estructura organizacional y los fondos utilizados en la realización de esas mismas transformaciones; (b) El proceso decisional, que es un proceso de transformaciones de información, bajo la forma de redes de conversaciones incesantes, que permiten activar y desactivar procesos, regularlos, y coordinarlos permanentemente a fin de mantener las variables de esos procesos dentro de límites soportables por el sistema, al mismo tiempo que esas cadenas de transformaciones permiten la producción de los bienes y/o servicios que constituyen la actividad principal de la organización en relación a su nicho; (c) El proceso de estructuración, el que va generando la estructura total que soporta los procesos ya mencionados mediante la producción fractal de parejas estructurales básicas. Estas parejas están compuestas de dos módulos: uno llamado módulo tecnológico (que realiza las transformaciones requeridas) y el módulo de pilotaje encargado de activar regular, y coordinar las transformaciones realizadas por el módulo tecnológico. Los módulos de pilotaje están integrados configurando en la unidad de interacciones, el sistema decisional. Los módulos tecnológicos también están integrados en la trama de relaciones que permite configurar el sistema estructural de ese sistema complejo (Mélèse, 1991; Arnold et al., 2011).

Es así que, en el contexto del sistema educacional chileno y la realidad regional de la Universidad Arturo Prat, a través de las carreras de Ingeniería que pertenecen a la Facultad de Ingeniería y Arquitectura, se desarrolla una aproximación hacia un Modelo de Gestión para la Docencia Universitaria.

\section{METODOLOGIA}

Se desarrolla una investigación de estudio de casos en una institución de Educación Superior del estado de Chile: Carreras de Ingeniería de la Universidad Arturo Prat-Chile.

La Facultad de Ingeniería y Arquitectura (FIA), desarrolla el ejercicio de una autoevaluación en el 2010 y durante los procesos de acreditación de las carreras de Ingeniería Civil: Ambiental (ICA), Metalúrgica (ICME), Industrial (ICl) y de Computación e Informática (ICCI), de la Universidad Arturo Prat de Chile (2011), se 
detectan una serie de fortalezas y debilidades, destacando negativamente la deserción en primer año, la cual está por sobre el $40 \%$, veinte puntos sobre la media nacional afectando los años de acreditación de ICA 2 años, ICME 3 años, ICCI 4 años e ICI 5 años. Lo anterior, motiva una revisión de todas las carreras de la Facultad y se inicia un trabajo en el que se establecen dos líneas de acción, una que permita desarrollar actividades para evaluar factores críticos que inciden en el proceso formativo y, una segunda línea de acción, que busca desarrollar indicadores de gestión docente, que permitan realizar seguimiento e intervenciones oportunas considerando el contexto educacional.

Desde la primera línea de acción se trabaja un rediseño curricular en las carreras de ingeniería: perfiles basados en competencias (Icarte, 2016), acortamientos de las carreras de ingeniería a 5 años, un tronco común con 18 actividades curriculares, incorporación de los sistemas de créditos transferibles (SCT), creación de dos carreras nuevas civil de Minas y civil Electrónica y, un proceso reflexivo para definir hacia dónde avanzar considerando tanto el Plan Estratégico, como el Modelo Educativo Institucional centrado en el estudiante. Estas acciones dan pie y son la base de este trabajo que se inicia el 2010, con un plan de trabajo y se materializa el 2013, con la implementación del correspondiente rediseño de las Ingenierías. Entre los años 2014 y 2015 se apalancan recursos a través de un proyecto Mecesup UAP1402, con un financiamiento de M\$270.000 del Ministerio de Educación chileno equivalente (unos 483 mil dólares americanos a marzo de 2014), que permite concretar intervenciones de ajustes microcurriculares, conocer otros casos de éxito nacionales e internacionales, mejorar infraestructura y consolidar tanto un equipo docente como de gestión con competencias pertinentes a los nuevos desafíos.

Para el desarrollo del Modelo de gestión en docencia se utiliza la metodología de Redes Modulares o Caja Negra (Análisis Modular de Sistemas), representada en la figura 1, se debe realizar la identificación de la organización y estructura decisional del sistema bajo análisis, y su despliegue de recursividad (niveles de gestión), asociados a los procesos. Para modelar se debe identificar el Proceso Primario o esencial, que va en directa relación con el propósito organizacional y que le otorgan sus elementos únicos y diferenciadores (identidad de clase). Posteriormente, se deben identificar los procesos decisionales, que corresponden a los procesos que estructuran y dan forma al funcionamiento de la organización.

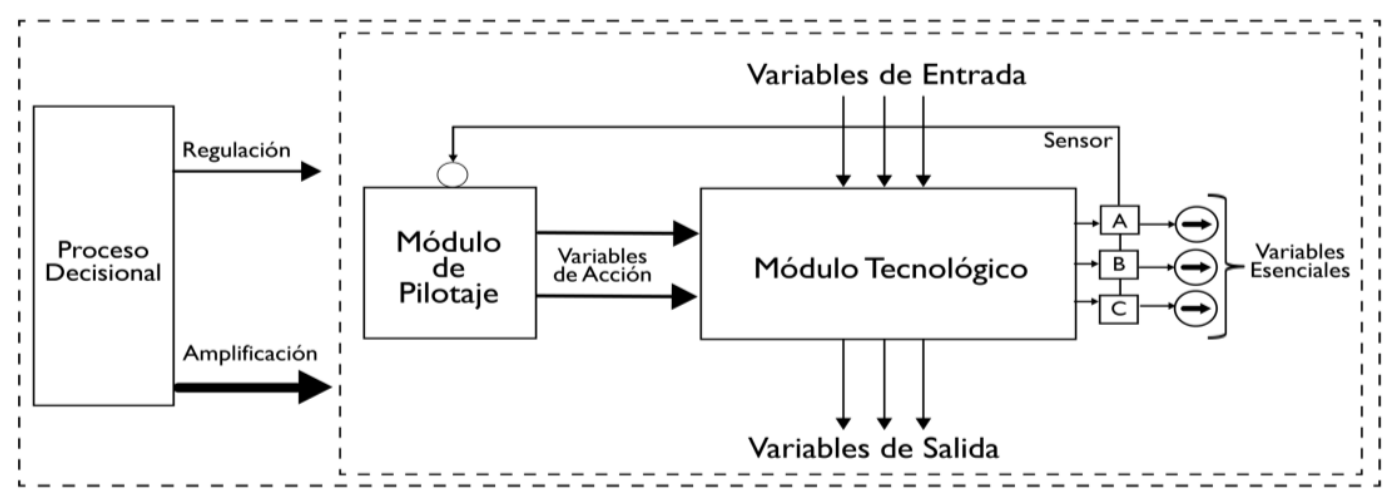

Fig. 1: Red Modular Genérica (Limone y Cademártori (1998), Plaza y Opazo (2015) basado AMS de Jacques Mélèse 1991)

Dentro del proceso decisional se identifican Módulos o Cajas Negras, que corresponden al escenario en el cual se realizan los ciclos conversacionales, que implican las tomas de decisiones y la ejecución de las acciones, y que gatillan las transformaciones asociadas a la ejecución de todos los procesos que impulsan y mantienen la organización, la cual se encuentra identificada como el Proceso Primario. Los módulos a los que se hace referencia son los siguientes: Módulo de Pilotaje y Módulo Tecnológico.

El Módulo de Pilotaje, representa en el modelo al ente activador de las acciones y en su rol de gestor contribuye a la regulación y coordinación de los módulos asociados a los procesos decisionales. El Módulo Tecnológico es un ente ejecutor de la acción, condicionado a las regulaciones y coordinaciones del módulo de pilotaje. Dentro de este nivel es posible reconocer las variables esenciales, las cuales permiten visibilizar el desempeño en las transformaciones asociadas a la ejecución de las decisiones. Además, se puede mencionar que tanto la activación de las acciones, a través de decisiones, como la ejecución de ellas, se realiza considerando una serie de insumos, los cuales corresponden a lineamientos organizacionales como planes estratégicos, políticas, reglamentos, normativas, entre otros, información de tipo interna y/o externa, para luego generar salidas, que es información que activa otros procesos. Todo lo anterior está orientado al logro de las variables esenciales, lo que permite su trazabilidad y medir el impacto de las acciones implementadas, en base a las decisiones determinadas por quien cumple el rol de gestor. Para este estudio de caso, se identifican las variables esenciales en base al Análisis de Progresión del Estudiante. 
El Análisis de Progresión del Estudiante corresponde a una secuencia de etapas que permite dar coherencia a las acciones y decisiones asociadas a la gestión académica, en función de procesos e indicadores de desempeño (variables esenciales), enfocándose en el ciclo de vida del estudiante en la Universidad, partiendo con la selección y matrícula de primer año, siguiendo con la deserción y la aprobación, y terminando con la titulación y la empleabilidad, como se presenta en la figura 2. El Ministerio de Educación en Chile, ha instalado el concepto de indicadores de la progresión, tales como: de deserción/retención en primer año y de niveles superiores así como deserción acumulada de segundo y tercer año; de reprobación/aprobación anual, por nivel; tiempo promedio de permanencia en la carrera, titulación oportuna los cuales son evaluados en los procesos de acreditación.

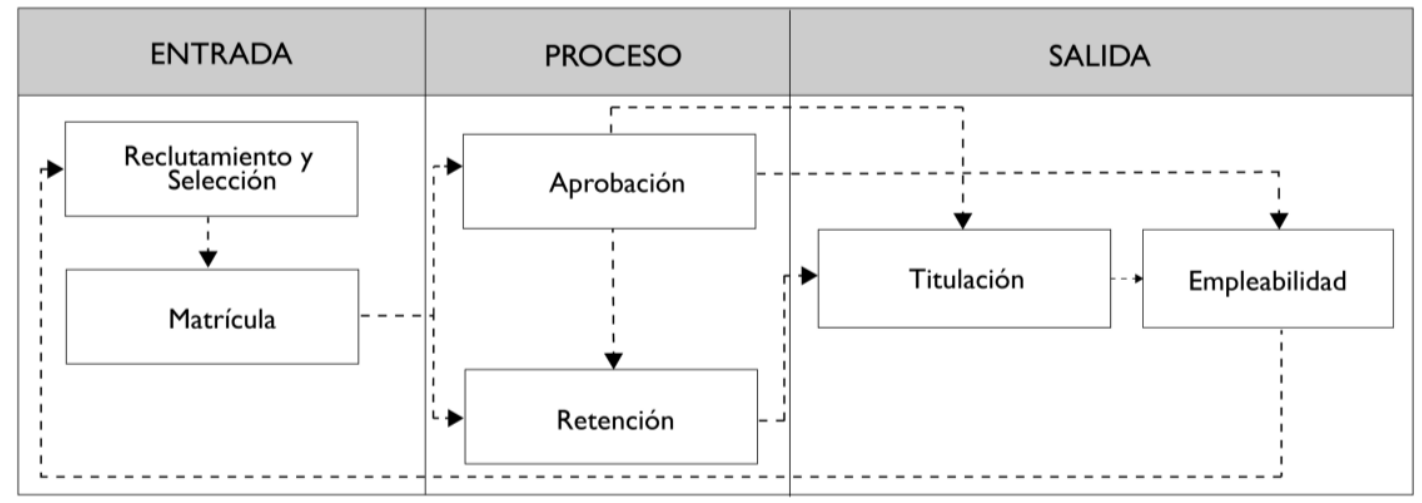

Fig. 2: Análisis de Progresión del Estudiante (Reproducida con autorización de Plaza y Opazo 2015).

Este método permite mantener visibles y bajo control indicadores de resultado asociados a todo el proceso de progresión académica del estudiante manteniendo en perspectiva y de manera equilibrada en todo este ciclo de vida una retroalimentación y conocimiento acerca del desempeño de la Carrera, la Facultad o la Institución. Este concepto permite identificar las etapas en los procesos docentes y reconocer los módulos decisionales.

Para el desarrollo del Modelo de Gestión se realizan una serie de actividades relacionadas con el levantamiento de información asociada a los procesos decisionales de la Facultad de Ingeniería y Arquitectura. Se planifican actividades de difusión y retroalimentación de resultados asociados al modelo referencial de gestión efectiva de carreras (Plaza y Opazo, 2015), para la sensibilización e instalación del modelo en los directivos y la utilización de los sistemas de información necesaria para su implementación. Como estrategia de implementación se utiliza como referencia el modelo SECl (Nonaka y Takeuchi, 1995) para generar espacios conversacionales en distintos niveles (ontología), con sus respectivos contextos y propiciar la interacción del conocimiento tácito y explícito, por medio de cuatro formas de conversión que es la socialización, exteriorización, combinación e interiorización.

Dentro del proceso de recopilación de datos, difusión de información y sensibilización de instrumentos y procedimientos, se realizan actividades presenciales en la Facultad, unidad de pilotaje, donde se revisan los instrumentos de recopilación de información, se analizan el marco conceptual asociado al modelo, se muestra el desarrollo de indicadores, se analizan resultados y se planifican actividades vinculadas a algunas acciones asociadas al modelo. También se llevan a cabo reuniones con unidades institucionales, para efectos de orientar recursos y planificar productos o reportes de la unidad hacia la Facultad de Ingeniería y Arquitectura. Para la consolidación del modelo propuesto se analizan los instrumentos formales de gestión que se utilizan tanto a nivel institucional como a nivel de Facultad, para lo cual se realiza una revisión de procesos decisionales y de sus parámetros, identificando elementos como Plan Estratégico Institucional, Plan de Desarrollo de la Facultad, Modelo Educativo, Planes de Mejoramiento de Acreditación y Sistemas de Información Institucionales que son parte estructural del modelo propuesto.

\section{RESULTADOS}

Para introducir los resultados es importante aclarar que la implementación del Modelo fue gradual liderado en términos operativos por la Dirección de Docencia y desde el ámbito político y de poder por el Decano. Para ello se genera un plan de trabajo en base a reuniones/talleres individuales y grupales con los directores de las carreras de Ingeniería de la Facultad de Ingeniería y Arquitectura y con académicos de las carreras y ciclo de asignaturas comunes denominadas tronco común. En estas reuniones/talleres se discutieron principalmente el nivel de comprensión del modelo, las actividades relevantes emprendidas por cada carrera en función del modelo y las condiciones para mejorar su funcionamiento asegurando viabilidad. Se utilizan estrategias de reforzamiento positivo y acompañamiento por quienes lideran el proceso o expertos que se 
integran para expandir los paradigmas de quienes componen la facultad, para generar nuevas posibilidades de observación y permitir la construcción de nuevas realidades.

El diseño de los principales procesos del Modelo de Gestión Docente de la Facultad de Ingeniería y Arquitectura (FIA), en base al modelo genérico, son los siguientes: (1) Módulo de Entrada: Selección y Matrícula, (2) Módulo de Proceso: Aprobación y Deserción y (3) Módulo de Salida: Titulación y Empleabilidad.

De acuerdo a la metodología expuesta, a continuación se muestran los resultados del Modelo de Gestión Docente FIA, representados en las figuras 3, 4 y 5 que pueden integrarse constituyendo el Modelo Integral a nivel de la Facultad.

La figura 3, muestra la estructura del primer módulo o subsistema de entrada del modelo de gestión propuesto, asociado a las decisiones relacionadas con el proceso de selección y matrícula para primer año. Los principales procesos asociados a este módulo corresponden a la Vinculación con Postulantes y la Incorporación de Estudiantes nuevos, procesos que se realizan dentro de un marco Institucional y particular a la carrera. Respecto del primero, se encuentran la Estrategia Corporativa con restricciones emergentes del Ministerio de Educación y el Modelo Educativo, y respecto del segundo, se encuentran el Plan de Desarrollo de la Facultad, los planes de mejoras relacionados con la Acreditación de Carreras y políticas específicas.

Adicionalmente y de manera transversal, se considera el Seguimiento de Estudiantes, que se realiza a nivel institucional a través de la Unidad de Análisis Institucional y por la Facultad, a través de la unidad de docencia. Se gatillan acciones por los directores de carrera apoyados en general por: estudios de preferencias de estudiantes de cuarto medio generados por la Dirección de Admisión, estudios de empleabilidad aportados por la oficina de Egresados y titulados complementados por el SIES, procesos de autoevaluación recientes, estrategias regionales, convenios con colegios, alianzas con instituciones regionales del rubro minero, turístico, salud, educación, información histórica, entre otros, para consolidar los esfuerzos de la vinculación cerrando el ciclo con su ingreso. El equipo de Gestión de la FIA con todos los directores de carrera se reúne para analizar las postulaciones a penas se liberan, de tal forma de velar por un proceso de matrícula eficiente al captar a los estudiantes de primera preferencia por la UNAP.

En resumen, el primer proceso, de Vinculación con Postulantes, es activado en el Módulo de Pilotaje, internalizando la información externa proveniente de medios de comunicación (SIES, otros estudios) y la información corporativa enviada por la Dirección de Planificación y Análisis Institucional. Adicionalmente, el Módulo de Pilotaje considera la información del Perfil del Estudiante y las instancias de Coordinación y Despliegue de tareas (incluyendo académicos), quienes desarrollan las actividades de vinculación con postulantes. La ejecución de la acción de Vinculación con Postulantes es evaluada a través de indicadores de Postulaciones Efectivas por Vacante, \% de Seleccionados en 1 a Preferencia e indicadores de Calidad de la Selección (puntaje Prueba de Selección Universitaria (PSU), Promedio Puntaje de Selección (PPS), Notas Enseñanza Media (NEM)), resultados que son parte de los indicadores de Progresión del Estudiante, manejados centralizadamente en la Institución a través del Sistema Ejecutivo de Indicadores Corporativos (SEIC).

La figura 4, muestra la estructura del segundo módulo o subsistema de proceso del modelo de gestión, asociado a las decisiones relacionadas con el proceso de Aprobación y Deserción. En él se observa que los principales procesos relacionados a este módulo corresponden a la ejecución de las Tutorías y Remediales y la Vinculación con Estudiantes en Riesgo de Abandono, al igual que el módulo anterior, estos procesos se realizan dentro de un marco Institucional considerando lineamientos Corporativos (Vicerrectoría Académica, Dirección General de Docencia) y otro particular a cada carrera incluyendo las mismas políticas ya mencionados.

En términos generales, como resultado de la acción de Incorporación de Estudiantes de 1er Año, se establece el Perfil del Matriculado, información que, en conjunto con las acciones de coordinación inter carreras proveniente del proceso siguiente, los resultados de la Encuesta de Compromiso Estudiantil y los Módulos con Alta Reprobación, activan el Módulo de Pilotaje asociado al proceso de Tutorías y Remediales. A partir de estos elementos, el Módulo de Pilotaje considera la información del porcentaje de reprobación y las notas promedio On-Line, más las instancias de Coordinación y Despliegue de tareas, las cuales incorporan a académicos, estudiantes y profesionales (diseño de instrumentos para identificar competencias de ingreso, programa de reforzamiento de competencias iniciales, innovación en docencia, diseño de tutorías virtuales y presenciales, comunidades de aprendizaje, entre otras). La ejecución de la acción de Tutorías y Remediales es evaluada a través de indicadores de Aprobación/Reprobación Promedio por Estudiante de 1ํㅜ $2^{\circ}, 3^{\circ}, 4^{\circ}$ y 5 Nivel, resultados que son evaluados a nivel de unidad después de cada periodo de evaluación y centralizadamente en la Institución a través de los Sistemas de: Alerta Temprana (SAT) y SEIC. Como resultado de la acción anterior, se establecen las prioridades para vinculación, priorizando a los estudiantes 
de bajo desempeño, se identifican los estudiantes que siguen procesos académicos normalmente y se distinguen las instancias de coordinación inter carreras. El Ejecutivo de la Progresión que pertenece a la unidad de docencia de la Facultad retroalimenta el proceso de Incorporación de Estudiantes de primer año y a los directores de carrera con requerimientos específicos así como estudios de caracterización de la deserción, Encuesta de Compromiso Estudiantil de estudiantes antiguos, entre otros.

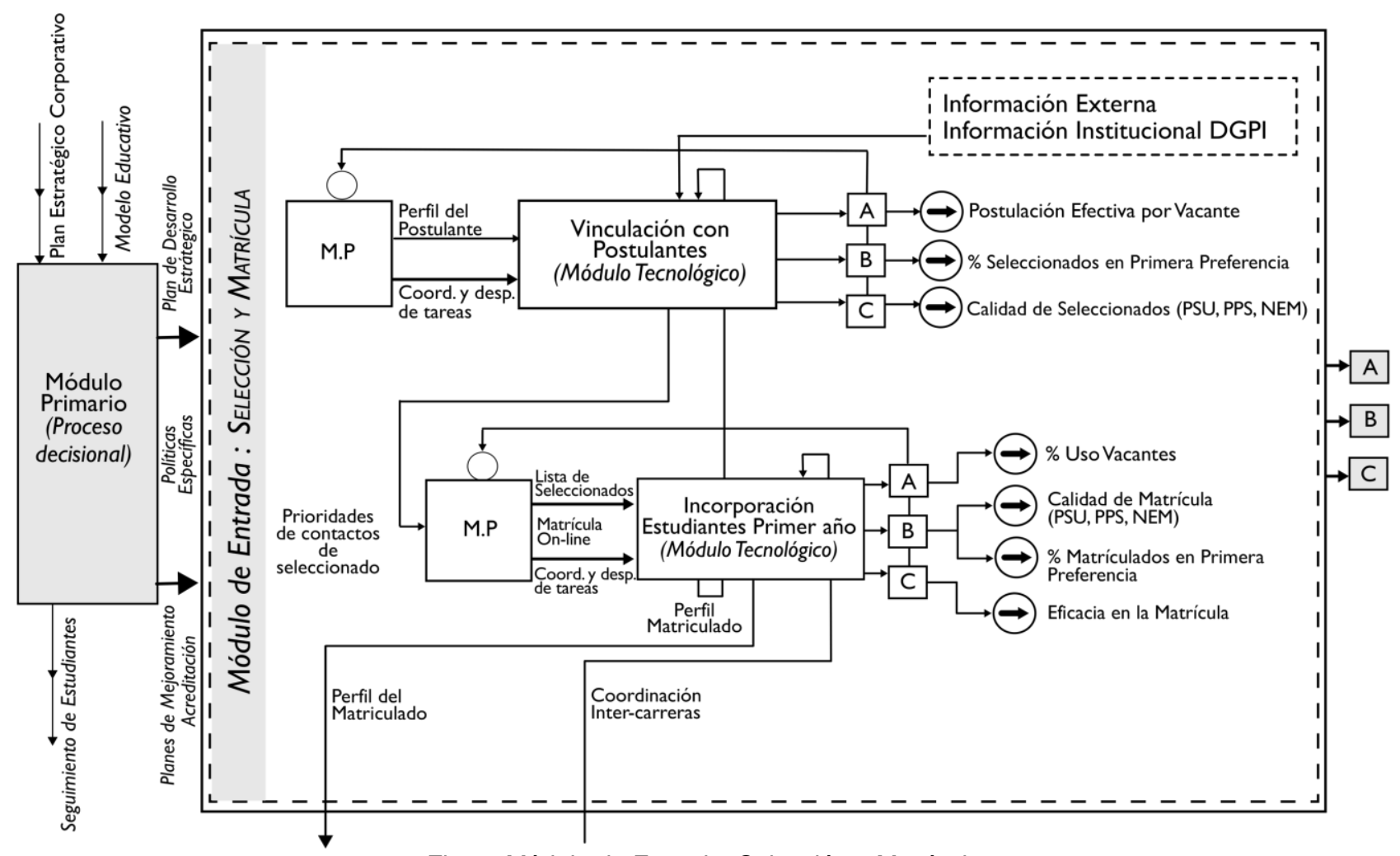

Fig. 3: Módulo de Entrada: Selección y Matrícula

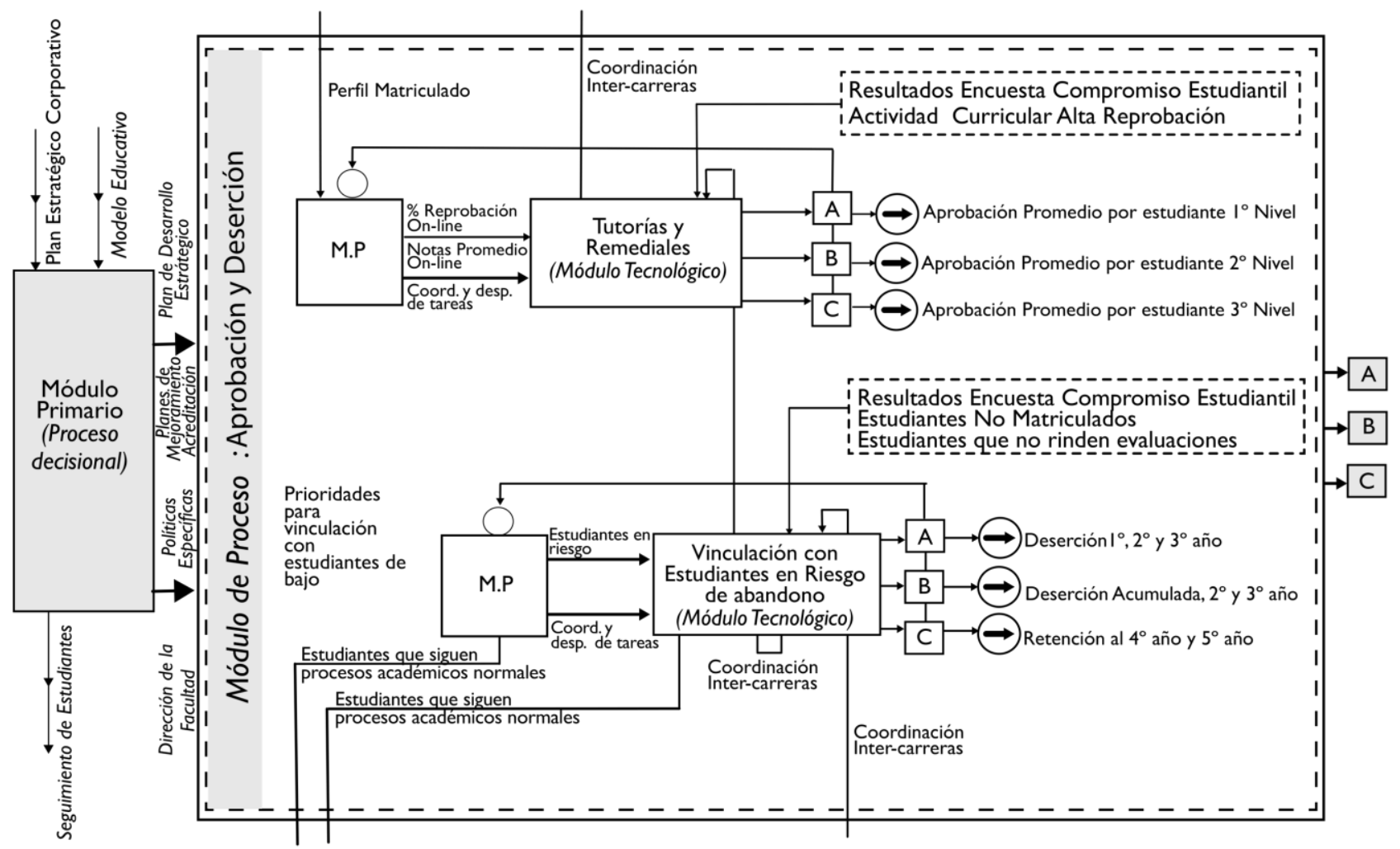

Fig. 4: Módulo de Proceso: Aprobación y Deserción 
Consecuentemente, dadas las prioridades para la vinculación con estudiantes de bajo desempeño, en conjunto con las acciones de coordinación inter carreras proveniente del proceso siguiente, los resultados de la Encuesta de Compromiso Estudiantil, la información de estudiantes no matriculados y la información de estudiantes que no rinden evaluaciones, se activa el Módulo de Pilotaje asociado al proceso de Vinculación con Estudiantes en Riesgo de Abandono.

A partir de estos elementos, el Módulo de Pilotaje considera la información de Estudiantes en Riesgo, proveniente de los estudios de caracterización de la deserción y del Sistema de Alerta Temprana, y las instancias de Coordinación y Despliegue de tareas, las cuales incorporan a académicos, gestores, estudiantes y profesionales. La ejecución de la acción de Vinculación con Estudiantes en Riesgo de Abandono es

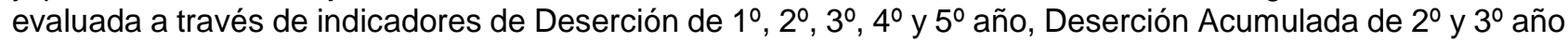
y Retención de $4^{\circ}$ año, resultados que también son desarrollados por SEIC y complementados con información de la unidad de docencia de la FIA. Como resultado de la acción anterior, se identifican los estudiantes que siguen procesos académicos normalmente y se distinguen las instancias de coordinación inter carreras por parte del Ejecutivo de la Progresión de la Facultad.

Este módulo permite a la unidad reconocer la necesidad de mantener estudios de investigación con respecto al fenómeno de la deserción para alcanzar al menos la media nacional por carrera. En una primera aproximación sistémico-compleja, como el compromiso de estudiantes de primer año para promover el aprendizaje y la persistencia (Kahu, 2017), la relación de las percepciones de los estudiantes y la relevancia del plan de estudios (Zepke, 2015) e incorporar otra forma de analizar el éxito estudiantil, a través de los ojos de los estudiantes (Naylor, 2017). Sólo entonces las universidades pueden avanzar en el fenómeno de la deserción y reducir las brechas de equidad que plagan la sociedad (Tinto, 2017). En la actualidad la facultad ha desarrollado un modelo de caracterización de la deserción para primer, segundo y tercer año que se sustenta teóricamente en la progresión del estudiante y el modelo interaccionista de Vincent Tinto, para ello se han considerado instrumentos validados científicamente como la Encuesta de Compromiso Estudiantil para estudiantes nuevos y otro para estudiantes antiguos.

Además, del seguimiento y retroalimentación cuando se contextualizan los indicadores surgen espacios de reflexión y construcción (SECI) sobre la implementación del Modelo Educativo Institucional, centrado en el estudiante, con perfiles basados en Competencias que se traducen en Resultados de Aprendizajes en los Programas de Actividades Curriculares (PAC) y que deben ser coherentes con las metodologías de enseñanza-aprendizaje y de evaluación. Esto obliga a generar una espiral de aprendizaje continuo relevando la Innovación Docente al quehacer académico y articulado a indicadores de impacto en la progresión (Política de Docencia FIA que incorpora lineamientos y recursos para la innovación). Se vinculan indicadores de innovación (tasa de PAC con micro ajustes por una comunidad de aprendizaje) con indicadores de progresión (tasa de aprobación); cantidad de proyectos de innovación docentes anuales. Se divulga la innovación docente en la Facultad, a través de un Seminario anual a cargo de la Dirección de Docencia FIA, para compartir experiencias al interior de la unidad e invitando a otras Facultades vinculadas.

La figura 5, muestra la estructura del tercer módulo o subsistema de salida, asociado a las decisiones relacionadas con el proceso de Titulación y Empleabilidad, que corresponden de manera general, al Seguimiento a Estudiantes de Último Año y la Vinculación con el Entorno Profesional, al igual que el módulo anterior, estos procesos se realizan dentro de un marco Institucional y otro específico a la Facultad con cada carrera (compromisos de acreditación).

Continuando con la descripción del modelo, en el módulo de Titulación y Empleabilidad se observa que, como resultado de la acción de Tutorías y Remediales y la acción de Vinculación con Estudiantes en Riesgo de Abandono, se identifican los estudiantes que siguen procesos académicos normalmente, información que activa el Módulo de Pilotaje asociado al proceso de Seguimiento a Estudiantes de Último Año.

A partir de estos elementos, el Módulo de Pilotaje considera la información Estudiantes de último año y el flujo en la titulación, más las instancias de Coordinación y Despliegue de tareas, las cuales incorporan a académicos, profesionales y la unidad de docencia (reportes intermedios con avances curriculares por estudiante en relación a una cohorte, identificación de anomalías administrativas que inciden en la titulación oportuna, gestión de procesos de titulación: diversificación, difusión de reglamentación de titulación y mecanismos efectivos de apoyo). La ejecución de la acción de Seguimiento a Estudiantes de Último Año es evaluada a través de indicadores de Porcentaje de Titulación Oportuna y Tiempos de Titulación, donde es evaluado centralizadamente en la Institución a través del SEIC, y también por la unidad de docencia de la Facultad. 


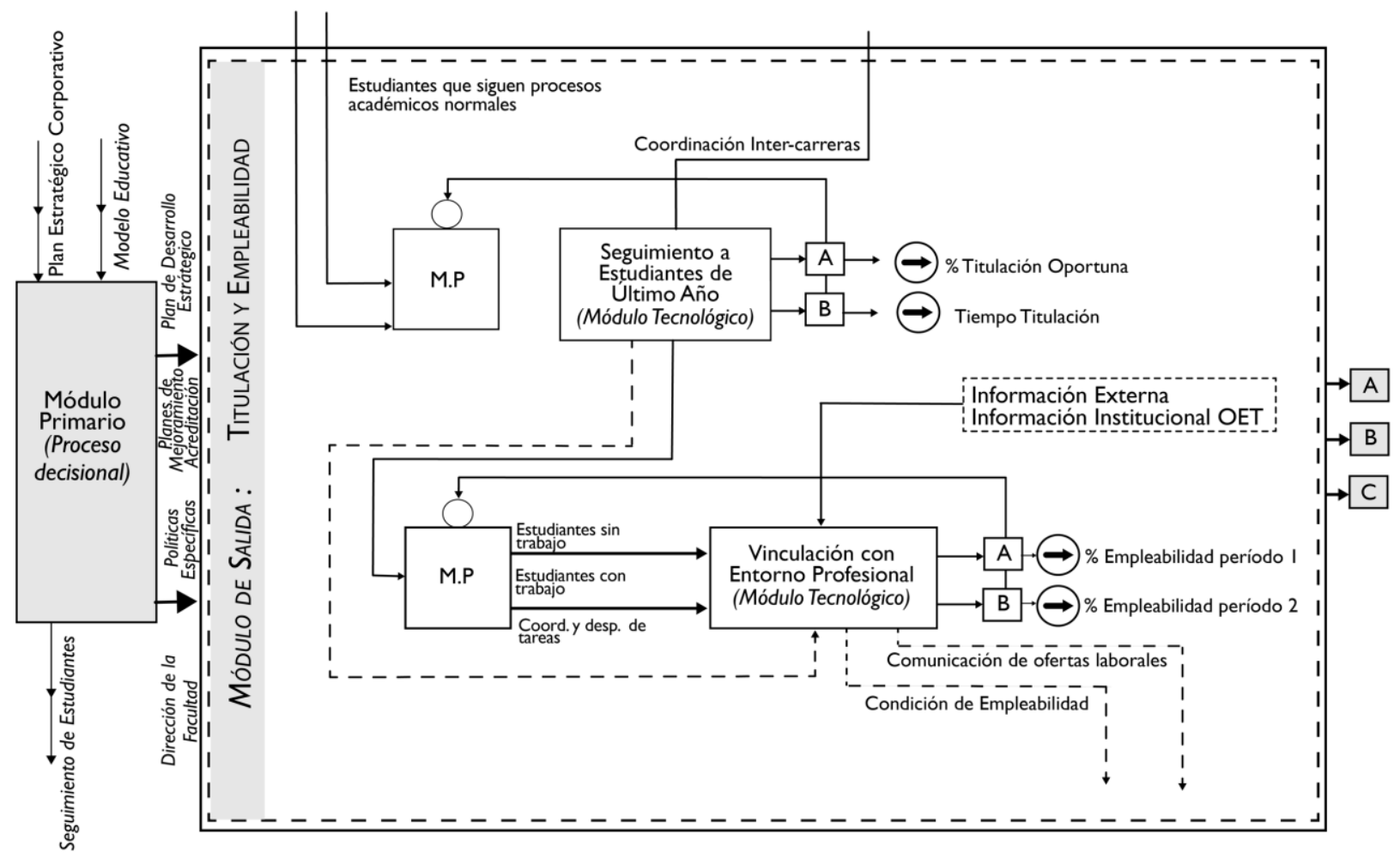

Fig. 5: Módulo de Salida: Titulación y Empleabilidad

Al analizar las acciones de Vinculación con el Entorno Profesional, el modelo propone la identificación de estudiantes sin trabajo y estudiantes con trabajo (a través de mecanismos formales y sistemáticos), los cuales permitirán realizar gestiones tendientes a reconocer las condiciones de empleabilidad de los profesionales egresados y la gestión de ofertas laborales (estudios de fuentes externas como el SIES). Las acciones asociadas a la Vinculación con el Entorno Profesional, están dirigidas a mantener bajo control los indicadores de Empleabilidad en diferentes condiciones de periodicidad. Actualmente, la Institución a través de la Oficina de Egresados y Titulados (OET) con su plataforma para el levantamiento de datos. Esta oficina integra la red de colaboración de oficinas de egresados y titulados o equivalentes del $\mathrm{CRUCH}$ (Consejo de Rectores de las Universidades Chilenas) cuya estrategia es generar esfuerzos mancomunados y compartir experiencias de éxito. Por otro lado, la Facultad se encuentran desarrollando planes sistemáticos e integrados orientados a gestionar información pertinente para la toma de decisiones asociadas a este proceso (desarrollo de instrumentos complementarios a la oficina de egresados, programa de fidelización, revisión de casos de éxito, retroalimentación del proceso formativo, levantamiento de requerimiento de especialización disciplinar, entre otros estudios e investigaciones) por lo que su consolidación resulta de gran relevancia. Las consideraciones anteriores implican que el contar con el diseño de un modelo de gestión permite, generar las acciones necesarias para consolidar los instrumentos y mecanismos de coordinación externa con el mundo laboral, que permitan mantener bajo control y hacer gestión en estos procesos, los cuales implican una mayor complejidad, ya que se dan fuera de las fronteras de la Institución y por tanto, tienen características distintas. Este módulo retroalimenta el proceso formativo al detectar la pertinencia del perfil profesional en el ámbito laboral y sus nuevos requerimientos a nivel de pregrado, post grado y especializaciones.

La búsqueda del equilibrio entre lo objetivo y lo interpretativo genera un gran desafío en el ámbito de la gestión, en una Universidad Pública con Responsabilidad Social. Por muchos años el paradigma de los estudiantes malos y buenos inspiró decisiones a quienes eran responsables del proceso formativo. Los que se formaron en el rigor de superar grandes obstáculos para ser merecedores de un título Universitario, aunque se discursee que los tiempos han cambiado, aún hay un rezago de académicos con esa visión. Esto, los autores de este trabajo lo resaltan por lo que significa el impulsar el cambio al interior de una Facultad constituida por una heterogeneidad de individuos con filosofías y experiencias de vida diversas que colocaron a prueba los principios teóricos planteados del trabajo. Los cuales se disipan con la cibernética de segundo orden al abrir un espacio para la reflexión sobre el propio comportamiento entrando directamente en el territorio de la responsabilidad y la ética. El modelo permite contar con una estructura para orientar la discusión y reflexión en los ciclos conversacionales por los distintos observadores que son responsables de gatillar acciones y construir una realidad organizacional. Estos ciclos conversacionales desde el pensamiento complejo, según Morin, nos permite el surgimiento de las contradicciones, como una apertura al conocimiento nuevo, lejos de significar un insoluble, se convierte en sí mismo en un progreso del conocimiento. 
Antagonismo y complementariedad son dos polaridades de un mismo fenómeno, entre los cuales oscilan los procesos vivientes que se hacen y se deshacen y los bucles organizacionales unen sus oposiciones sin anularlas jamás (Tarride, 1995).

Se modeliza una propuesta con niveles recursivos que permite absorber la complejidad con las particularidades de la Facultad de Ingeniería y Arquitectura, que busca instalar una visión de gestión con mecanismos que permitan evidenciar situaciones o anomalías con orientación a desempeños. En la búsqueda de eficacia y eficiencia en el ciclo de vida del estudiante en la Universidad (Progresión del Estudiante). Acompañada de una visión de Responsabilidad Social, declarado en la misión institucional, al comprender que es la responsabilidad de la facultad y carrera hacerse cargo de un ser humano con expectativas y esfuerzo personal/familiar en donde el compromiso es entregar las mejores condiciones para alcanzar un proceso exitoso. Para lo cual, se realizan evaluaciones en términos de análisis de variables esenciales del Modelo (indicadores de progresión) y variables de acción, lo que sucede en el aula, los efectos del diseño curricular, infraestructura, relaciones humanas internas, perfiles de ingreso, entre otros, en la formación de los estudiantes permitiendo una nueva visión con respecto a la dinámica de la docencia en la facultad. Esto permite enfocar la atención en lo relevante y concreto para explorar y buscar soluciones con equipos de individuos/observadores que son parte de la organización (directores de carrera, académicos, profesionales, administrativos, estudiantes, autoridades de la facultad, otros), para diseñar propuestas de solución que permitan intervenciones culturalmente factibles y sistémicamente deseables a nivel de facultad, carrera, tronco común y actividades curriculares.

La instalación de la cultura de la innovación al interior de la Facultad, se fue cimentando a través de las instancias reflexivas de los involucrados, en espacios de confianza construidos como parte del diseño (SECI) y guiados por el modelo de gestión.

Una intervención emblemática, fue la identificación de la variable comuna de Alto Hospicio como factor de riesgo, considerando que un tercio de los estudiantes de primer año de distintas cohortes provienen de esta comuna, que teniendo buen rendimiento académico, igual desertaban cuya problemática es la alta congestión para el traslado automotriz en las horas de la mañana (entrada al trabajo) y la tarde (salida del trabajo), lo que condicionaba el retraso de los estudiantes y por lo tanto ausencia en las clases, considerando que sus docentes con el ánimo de formar profesionales responsables no le permitían el ingreso al aula. A través de grupos focales realizados con estudiantes de Alto Hospicio, se verificó que, en la mayoría de los casos, existían variables externas que afectaban sus retrasos y ausencias. En virtud de la información se piloteó un horario para estudiantes de primer año a partir de las 9:00 horas, con bloques comprimidos (sin bloques vacíos), espacios para poder estudiar o esperar clases, el primer impacto fue aumento de la asistencia a clases sobre el $85 \%$ y mejora en la tasa de aprobación de las asignaturas de matemáticas involucradas, constituyéndose como política de Facultad. El impacto de esta intervención, es que desaparece la variable de vivir en la comuna de Alto Hospicio como factor de riesgo de deserción.

Otras acciones desarrolladas por las carreras, producto de brechas detectadas por el modelo, se canalizaron a través de proyectos los cuales corresponden a la utilización de mecanismos de Vinculación con Postulantes (proceso 1), específicamente diseñados por la carrera, que se traduce en mejoras significativas respecto de la calidad de la matrícula de primer año (postulaciones en primera opción, aumento de la masa crítica de postulantes, mejora en la cobertura de vacantes). Mejora de los procesos de inducción de estudiantes nuevos por parte de cada carrera y posteriormente la creación de mecanismos de acompañamiento como: Apadrinamiento de estudiantes antiguos a estudiantes nuevos, Programa de Tutores FIA con un acompañamiento integral con impacto en la retención de primer año. En el módulo 2, se han activado comunidades de aprendizajes para el rediseño, ajustes curriculares e innovación docente con incorporación de las tecnologías considerando los perfiles de las nuevas generaciones. Detección de instalar nuevas competencias en los docentes para seguir avanzado en nuevas prácticas e innovación en docencia, revisar y actualizar el perfil docente por niveles ya que incide en el clima de aprendizaje en el aula, entre otros.

En síntesis, los módulos que se han abordado en su totalidad son los Módulo de Entrada: Selección y Matrícula y Módulo de Proceso: Aprobación y Deserción, reconociendo que los escenarios son dinámicos y por lo tanto el modelo debe estar en un ajuste constante (mejora continua). El Módulo de Salida: Titulación y Empleabilidad, se aborda desde el seguimiento de los estudiantes que se encuentran en condiciones de titularse oportunamente para que se gestionen problemas que se puedan generar de tipo administrativo. Este módulo representa un desafío ya que se encuentra en etapa de implementación considerando que la cohorte 2013 (rediseño curricular), está ad portas de finalizar su plan de estudio. En la figura 6, se visualizan los impactos concretos que evidencian mejoras en indicadores de retención y aprobación de primer año en carreras de ingeniería, considerando como base el año 2010, sin modelo. Su aplicación con una gestión proactiva en docencia incide en el aumento de la titulación oportuna y transitar de no tener carreras de ingeniería acreditadas, a tener un promedio de 5,5 años de acreditación (de un máximo de 7 años). 


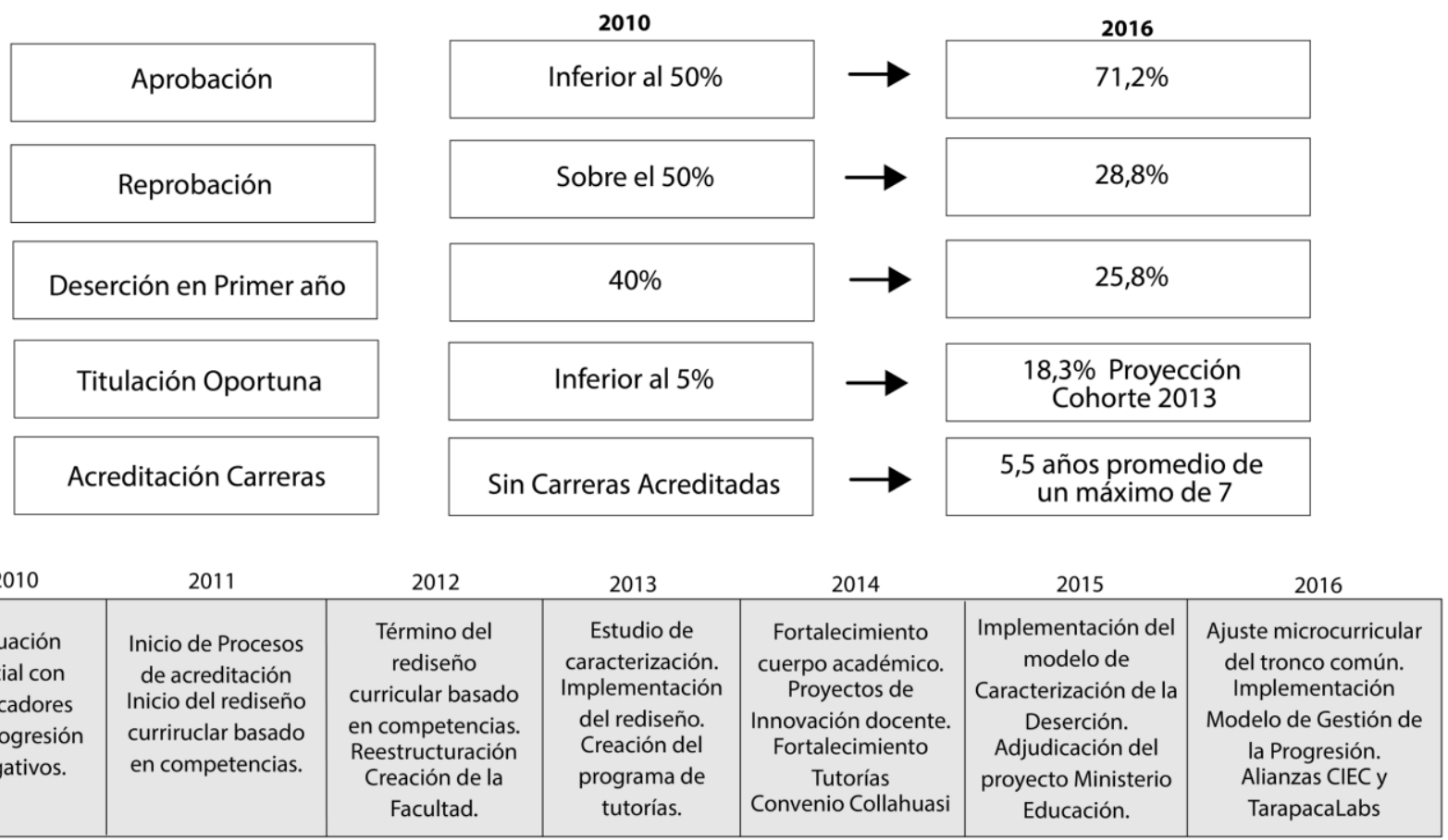

Fig. 6: Resultados comparativos de la Facultad de Ingeniería y Arquitectura período 2010 y 2016 . Unidad de Docencia Facultad de Ingeniería y Arquitectura / Unidad de Análisis Institucional

\section{CONCLUSIONES}

El Modelo de Gestión en Docencia Universitaria implementado ha permitido ordenar, sistematizar y orientar la gestión hacia desempeños en indicadores docentes. Además, de instalar procesos de detección de posibles anomalías con resolución proactiva que tiende a mejorar el proceso formativo. Este constructo se puede entender como un dispositivo sistémico-cibernético o como un dispositivo de Aprendizaje-Acción desde la Gestión del Conocimiento. Su puesta en marcha, como un piloto a nivel institucional, en la Facultad de Ingeniería y Arquitectura de la Universidad Arturo Prat con seguimiento del Ministerio de Educación de Chile, el cual se encuentra en un $95 \%$ aplicado, considerando que los módulos 1 y 2 están en pleno funcionamiento mientras que el tercer módulo se encuentra en un $85 \%$ de su implementación.

El impacto de la articulación del modelo de gestión en las carreras de ingeniería ha contribuido a reposicionar a nivel de la zona macro norte y nacional su desempeño al evidenciar un incremento positivo en sus indicadores de progresión del estudiante, destacando un incremento de 14 puntos porcentuales en la retención y 21 puntos porcentuales en la aprobación de primer año al año 2016. Todo esto, ha influenciado a las autoridades de la universidad la decisión de decretar el modelo para la Facultad y de aplicarlo con sus ajustes respectivos a las otras facultades para su institucionalización. Este trabajo generó la de integración de los Sistemas Institucionales como fuentes de información en tiempo real para nutrir el modelo y también articular con fuentes externas como el SIES y otras para alimentar los distintos módulos. Un aspecto que no es trivial, es el requisito de profesionalizar el rol de los equipos de gestión, para ello se capacitaron los académicos y profesionales pertinentes para instalar las bases para el funcionamiento del modelo.

Finalmente, contar con una herramienta de gestión moderna en una universidad Estatal y regional en Chile permite la mejora continua y facilita la trazabilidad de su desempeño para el aseguramiento de la calidad y enfrentar los procesos de acreditación, ya que en el año base no se realizaba gestión. El éxito del modelo no sólo radica en contar con indicadores, sino en que ellos orientan las construcciones conversacionales para reflexionar, integrando el conocimiento de la experiencia interna e incorporando conocimiento externo cuando es pertinente, sobre brechas en un contexto que orientan la toma de decisiones, construyendo una cultura participativa y reflexiva con respecto a su quehacer, lo que obliga a salir de la zona de comodidad hacia un espacio de aprendizaje continuo para la adaptación y transformación de la Facultad, hacia una Organización Inteligente, para ello es relevante alinear eficientemente los procesos, tecnologías y personas, que es lo que permite crear e intercambiar conocimiento para contribuir a la generación de valor.

\section{REFERENCIAS}

Arnold, M., A. Urquiza y D. Thumala, The Reception of the Concept of Autopoiesis in the Social Sciences, Sociológica, 26(73), 87-108 (2011) 
Ashby, W.R., An introduction to cybernetics, Chapman y Hall Limitada (1961)

Barnett, R., Claves para Entender la Universidad en una Era de Supercomplejidad, 1ㄹ Ed., 1-256, Pomares (2002)

Beer, S., The Culpabliss Error: a calculus of ethics for systemic world, Systems Practice, doi: 10.1007/BF02557886, 10(4), 365-380 (1997)

Espejo, R. y A. Reyes, Organizational system: Managing Complexity with the Viable System Model, 1르. Ed., 1-264, Springer-Verlag Berlin Heidelberg (2011)

Espejo, R., The Footprint of Complexity: The Embodiment of Social Systems, doi: 10.1108/03684920410523643, Kybernetes, 33(3/4), 671-700 (2004)

Espejo, R. y M. Schwaninger, Organizational Fitness: Corporate Effectiveness through Management Cybernetics, $4^{\underline{a}}$ Ed., Campus, Frankfurt (1993)

Icarte, G. y H. Labate, Metodología para la Revisión y Actualización de un Diseño Curricular de una Carrera Universitaria Incorporando Conceptos de Aprendizaje Basado en Competencias, doi: 10.4067/S0718-50062016000200002, Formación universitaria, 9(2), 03-16 (2016)

Kahu, E., K. Nelson y C. Picton, Student interest as a key driver of engagement for first year students, Student Success, 8(2), 55-66 (2017)

Limone, A., Acoplamiento Mosig-Ciborga: Una Explicación Científica de la Empresa, Enefa, Talca, Chile (2002)

Limone, A. y D. Cademártori, La empresa: una red de transformaciones, Santiago de Chile, Editorial Jurídica ConoSur Limitada (1998)

Maturana, H. y F. Varela, De Máquinas y Seres Vivos: Autopoiesis: la Organización de lo Vivo, 6ª Ed., Universitaria, Santiago, Chile (2006)

Maturana, H., La Objetividad: Un Argumento para Obligar, 1-148, Jc Sáez Editor, Santiago, Chile (2004)

Maturana, H., El Árbol del Conocimiento: las Bases Biológicas del Conocimiento Humano, Madrid, Debate (1990)

Mélèse, J., L'analyse Modulaire des Systèmes, AMS: Une Méthode Efficace Pourappliquer la Théorie des Systèmes au management, 1-233, Les Éditions d'organisation (1991)

Morin, E., From the concept of system to the paradigm of complexity, Journal of social and Evolutionary Systems, 15(4), 371-385 (2004)

Naylor, R., First year student conceptions of success: What really matters? Student Success, doi: 10.5204/ssj. v8i2.377, 8(2), 9-19 (2017)

Nonaka, I. y T. Takeuchi, The knowledge-creating company: How Japanese companies create the dynamics of innovation, Oxford university press (1995)

Nonaka, I., G. Von Krogh y S. Voelpel, Organizational knowledge creation theory: Evolutionary paths and future advances doi: 10.1177/0170840606066312, Organization Studies, 27(8), 1179-1208 (2006)

Plaza, E. y P. Opazo, Effective Career Management Experience in a Chilean Public University, 9th international Technology, education and development conference, 5861-5868, Madrid, Spain, Marzo (2015)

Rodríguez, D. y J. Torres, Autopoiesis, The Unity of a difference: Luhmann and Maturana, doi:10.1590/S151745222003000100005, Sociologías, (9), 106-140 (2003)

Senge, P., A. Kleiner y otros cuatro autores, The dance of change: The challenges to sustaining momentum in learning organizations, Facilitation, AResearch y Applications (2001)

SIES, Principales Resultados Evolución Retención de Primer Año 2010-2014.: SIES, División de Educación Superior, Ministerio de Educación (2015)

Tarride, M. y R. Capstick, Sistemas de Control de Gestión de hospitales públicos: reflexiones para una cultura de autorregulación distribuida, revista chilena de salud pública, 12(3), 169-180 (2008)

Tarride, M., Complexity and Complex Systems. História, Ciências, Saúde-Manguinhos, 2(1), 46-66 (1995)

Tinto, V., Research and Practice of Student Retention: What next? Journal of College Student Retention: Research, Theory y Practice, 8 (1), 1-19 (2007)

Tinto, V., Reflections on Student Persistence, doi: 10.5204/ssj. v8i2.376, Student Success, 8(2), 1-8 (2017)

Zapata, G. y I. Tejada, Informe Nacional-Chile. Educación Superior y Mecanismos de Aseguramiento de la Calidad, CINDA (2016)

Zepke, N. Student engagement research: thinking beyond the mainstream. Higher Education Research $y$ Development, 34(6), 1311-1323 (2015) 
